\title{
Human-Health-Analysis Semantic Computing \& 5D World Map System
}

\author{
Yasushi KIYOKI $^{\mathrm{a}, 1}$, Koji MURAKAMI ${ }^{\mathrm{b}, \mathrm{c}}$, Shiori SASAKI ${ }^{\mathrm{a}}$, Asako URAKI ${ }^{\mathrm{a}}$ \\ ${ }^{\mathrm{a}}$ Graduate School of Media and Governance, Keio University, Japan \\ ${ }^{\mathrm{b}}$ SFC Research Institute, Keio University, Japan \\ ${ }^{\mathrm{c}}$ Prevent Science Co., Ltd
}

\begin{abstract}
Semantic space creation and computing are essentially significant to realize semantic interpretations of situations and symptoms in human-health. We have presented a semantic space creation and computing method for domainspecific research areas. This method realizes semantic space creation with domainoriented knowledge and databases. This paper presents a semantic space creation and computing method for "Human-Health Database" with the implementation process for "Human-Health-Analytical Semantic Computing". This paper also presents a new knowledge base creation method for personal health data for preventive care and potential risk inspection with global and geographical mapping and visualization in 5-Dimensional World Map System. This method focuses on the analysis of personal health and potential-risk inspection and realizes a set of semantic computing functions for semantic interpretations of situations and symptoms in human-health. This method is applied to "Human-Health-Analytical Semantic Computing" to realize world-wide evaluation for (1) multiparameterized personal health data, such as various biomarkers, clinical physical parameters, lifestyle parameters, other clinical/physiological or human health factors, etc., for health monitoring, and (2) time-series multi-parameterized health data in the national/regional level for global analysis of potential cause of disease. This Human-Health-Analytical Semantic Computing method realizes a new multidimensional data analysis and knowledge sharing for a global-level health monitoring and disease analysis. The computational results are able to be visualized in the time-series difference of the values in each place, the difference between the values of multiple places in a focused area, and the time-series differences between the values of multiple places to detect and predict a potentialrisk of diseases.
\end{abstract}

Keywords. Semantic Search, Semantic Computing, Medicine, Medical Data, Big Data, Biographical Data, Vital Data, Sensing, AI, Cyber-Physical System, Visualization, Data Mining, Warning, SPA, Sensing, Processing, Actuation, SDGs

\section{Introduction}

Semantic computing is a promising approach to realize Human-Health-Analysis and Health caring. We have proposed semantic computing methods with a multidimensional semantic space creation architecture representing domain-specific knowledge and databases [1][2][3]. We have designed a domain specific knowledge space creation process for our semantic computing. In this paper, we apply this

${ }^{1}$ Corresponding Author, Yasushi Kiyoki, Keio University, Endo 5322, Fujisawa, Kanagawa, Japan; Email:kiyoki@sfc.keio.ac.jp. 
semantic computing method to Human-Health-Analysis and Health Caring, as "Human-Health-Analysis Semantic Computing Method".

The main objective of this method is to realize a semantic-space creation process and a semantic computing environment for semantic interpretations of situations and symptoms in human-health. This method uses domain-specific knowledge resources in "Human-Health Database," and creates various semantic spaces, consisting of domain specific knowledge expressions. The variety of semantic spaces is derived from parameters which a Human-Health database designer defines in the semantic-space creation process. Those parameters are expressed in two categories: (1) expertizedlevel determining the expertized-level parameters of semantic space, (2) sub-domainlevel determining sub-domain-specific parameters for defining semantic subspaces consisting of 'sub-domain' features. By using these parameters, this method controls the scope of the analysis, according to the objectives of the semantic analysis and interpretations.

In our "Human-Health-Analysis Semantic Computing," domain-specific knowledge is formalized as a featured-vector matrix in a semantic space, with expertized-level and sub-domain-level parameters. The domain-specific knowledge for Human-Health-Analysis is utilized for domain-specific semantic space creation. The matrix data structure of the domain-specific knowledge is expressed in the HumanHealth semantic space, and human-health data are mapped on to this semantic space. The semantic computing is applied to this space and realizes semantic interpretations of situations and symptoms in human-health with the relationships between disease and patients.

We have presented a concept of "Semantic Computing System [1][2][3]" and "5 Dimensional World Map System" for realizing global environmental data-analysis, integration, search and visualization for various issues in the world-wide scope. The main feature of 5D World Map System is to realize a knowledge-base platform of collaborative work for users to perform a global analysis for sensing data in a physical space along with the related multimedia data in a cyber space, on multiple views of time-series maps based on the spatiotemporal and semantic correlation calculations [7][9][10][12]. This paper applies 5D-World Map System to human-health analysis and support.

The "Human-Health-Analysis Semantic Computing Method" with semantic computing realizes semantic computing for biologic data, such as height, weight, BMI, blood pressure, blood glucose, blood protein, urine metabolites, images, personal genes, mRNA, etc. in the "multi-dimensional-biologic-parametrized semantic-space". This method realizes semantic associative computing of semantic equivalence, similarity and difference between "multi-dimensional-biologic data". We apply this system to human-health analysis, as a new platform of human-health care and support.

\section{Human-Health-Analysis Semantic Computing}

\subsection{Human-Health Semantic Space Creation and Semantic Computing}

A semantic computing method [1][2][3] is applied to human-health databases to realize health-health analysis in earlier stages in disease, as shown in Figure 1. Although the survival rate of patients related to disaster and pandemic is improving due to the development of new treatments, a worldwide perspective suggests that the number of 
patients will continue to increase. There is no change in the situation where the foundation of a healthy healthcare measure is required. It has been proven in many diseases that the earlier treatment in the earlier stage makes better results in the survival rate of patients. The fact is that future disease-control is focused on prevention and early detection. This semantic computing focuses on health-level analysis in diseases and effects related to disaster and infection-spread.

Process-1: Semantic Space Creation of " $n$-dimensional health-analysis universe"

(Step-1-1) A set of " $m 1$ patient-data" is given as n-parameters (human biological data (various biomarkers, clinical physical parameters, lifestyle parameters, other clinical / physiological or human health factors, etc.), and defined as the "patientdata matrix, and each patient-data element is characterized by $n$ features. That is, an $m$ by $n$ matrix $\boldsymbol{M 1}$ is defined as "patient-data matrix" in the "orthogonal semantic space $S$ " as " $n$-dimensional health-analysis universe".

(Step-1-2) A set of " $m 2$ non-patient-data" is given as n-parameters (human biological data (various biomarkers, clinical physical parameters, lifestyle parameters, other clinical / physiological or human health factors, etc.), and defined as the "non-patient-data matrix, and each non-patient-data element is characterized by $n$ features. That is, an $m 2$ by $n$ matrix $\boldsymbol{M} 2$ is defined as "nonpatient-data matrix" in the "orthogonal semantic space $S$ " as "n-dimensional health-analysis universe".

Process-2: Context-specific distance computing in the context-dependently-selected subspace from the semantic space $S$ :

(Step-2-1) Specific parameters, corresponding to a specific-context to be analysed, are selected from $n$-parameters" in $\boldsymbol{S}$, and the context is characterized to make analysis in a context-dependent way.

(Step-2-2) A "selected subspace", from the orthogonal semantic space, is extracted to be analysed according to a context. The subspace is projected according to the given "context" from the semantic space $\boldsymbol{S}$, which are given as "context" represented by "subset of parameters.

(Step-2-3) The metric for computing the distance between data elements in $\mathrm{m} 1$ and $m 2$ is defined in the semantic space, and the highly-correlated data elements between $m 1$ and $m 2$, to the given "context" are extracted, as the distance in the selected subspace. 


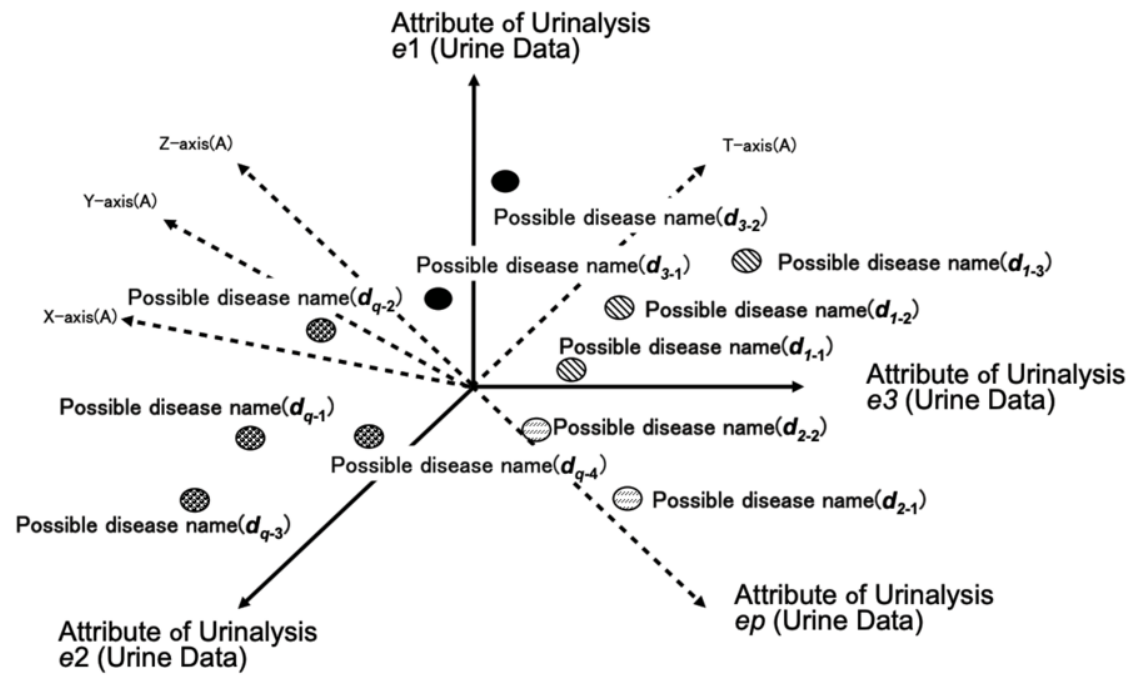

Possible disease names:Cancer / tumor, kidney disease, bladder / ureter / urethral disease, blood disease, heart disease, liver disease, pancreatic disease, hormonal imbalance.

Figure 1. Human-Health Semantic Space Creation for Semantic Computing

\section{5D World Map System for Human-Health-Analysis}

One of the important applications of the semantic computing system is "Human-Health Analysis," which aims to evaluate various situations and symptoms in human-health.

We have proposed and introduced a global knowledge sharing and processing system architecture as "5-Dimensional World Map System [4][6][8][13]," with multidimensional knowledge integration and visualization functions. This system has been applied to a lot of knowledge applications, as global environmental analysis and visualization. The basic space of this system consists of spatial $\left(1^{\text {st }}, 2^{\text {nd }}\right.$ and $3^{\text {rd }}$ dimensions), temporal ( $4^{\text {th }}$ dimension) and semantic dimensions $\left(5^{\text {th }}\right.$ dimension, representing a large-scale and multiple-dimensional semantic space that is based on our semantic computing system $([1][2][3][4])$. This space memorizes and recalls various multimedia information resources with temporal, spatial and semantic correlation computing, and realizes a 5D World Map for dynamically creating temporal-spatial and semantic multiple views applied for various "environmental multimedia information resources [9][10][12]"

As an important application of 5-Dimensional World Map System, we have constructed "5D World Map System for Human-Health-Analysis" for globally sharing and analyzing human health situations with semantic computing functions, applied to "human health data sharing," as a new platform of collaborative humanhealth analysis [4][6]. This platform enables to create a remote, interactive and realtime human health and academic research exchange among different areas. 


\subsection{D World Map System for Integration of Spatiotemporal and Semantic Computing}

In the design of this global human-health analysis system, we focus on how to search and analyze human health data related to human health situations, according to contexts and analysis-points.

"5D World Map System for Human-Health-Analysis" is structured, as shown in Figure 2, for sharing and analyzing human health situations and stimulus with semantic functions applied to "human health databases," as a new platform of collaborative human-health analysis.

We have already had track records in medical applications. We focus on simple urine sampling screening based on the biological characteristics of cancer, and realize new risk presentation methods by analyzing indices related to multiple parameters in a multidimensional manner. Urine specimens do not cause pain during collection and do not carry the physical risk of exposure to radiation for measurement. Based on these measurement results, we introduce the analysis concept of the 5D World Map, which is also used for regional disaster prediction, and succeeded in developing a new risk prediction. We propose a new concept of disease risk prediction by fusion of biology and information technology.

This system is constructed by SPA (Sensing-Processing-Actuation) process concept as shown in Figure 2.

The essential features of the system are described as followings:

(1) The system sets human biological data (various biomarkers, clinical physical parameters, lifestyle parameters, other clinical / physiological or human-health factors, etc.) as a target data and represents them as a $n$-dimensional matrix.

(2) The system sets the origin of each parameter as a neutral "semantic origin point" and represents the $n$ types of parameters as an $n$-dimensional space to measure the similarity of $n$ dimensions comprehensively and dimensionally by aggregating the parameter values at the origin for multiple parameters.

(3) The system calculates the distance in the $n$-dimensional space among the analysis subjects, the existing diseased patients and the healthy subjects as the disease occurrence risk degree of the subjects.

(4) The system visualizes and represents the risk of disease occurrence of the subjects as a Risk Person Discovery Method device using the graph of each dimension value as a polygon.

While the general existing methods in medical field grasps the tendency of the entire subjects' data distribution by statistics, this method realizes more detailed and fine-grain prediction and diagnosis because of the features described above. As described in the next section, the system consists of 3-phase checking process: $1^{\text {st }}$ Phase: Finding similar person with principal parameters, $2^{\text {nd }}$ Phase: Rule-based analysis with secondary-essential parameters, and $3^{\text {rd }}$ Phase: Time-series analysis. 


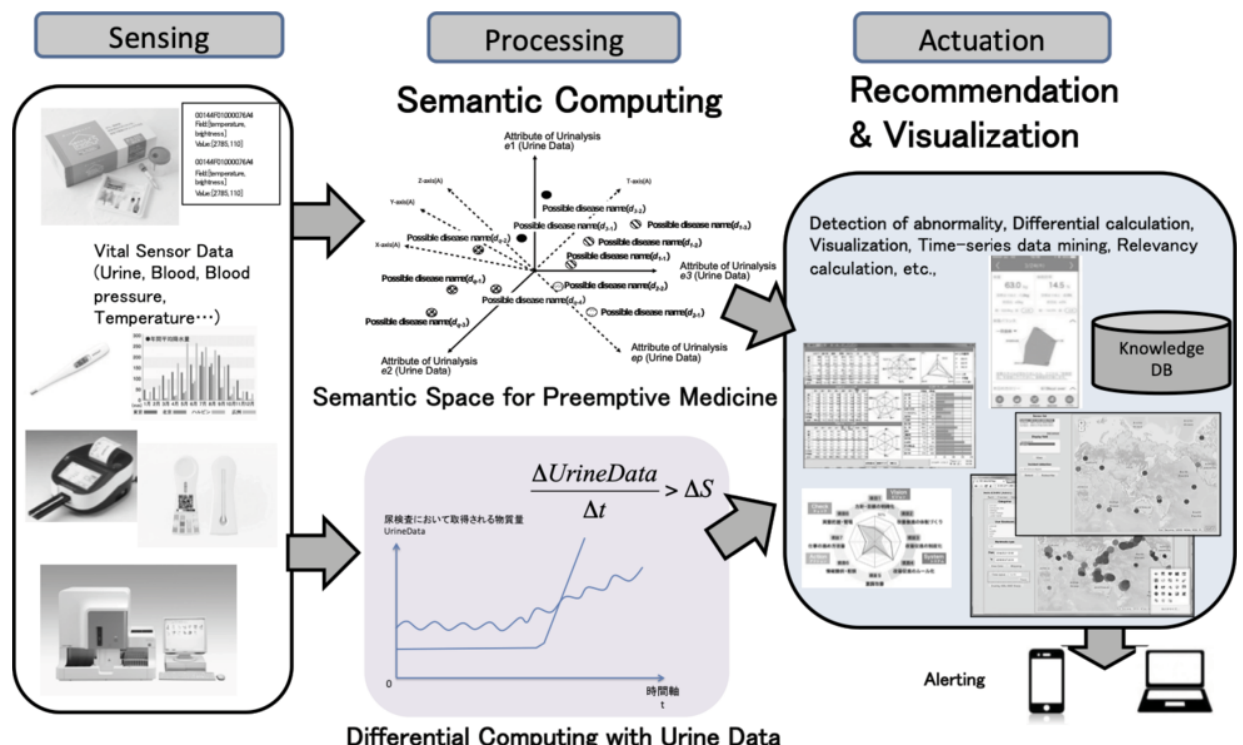

Figure 2. SPA process of 5D World Map System for Health analysis with semantic computing and visualization

5D World Map System [4][6][8][13] has been providing various functionalities to share and visualize various types of multimedia data [8][11][13]. A combination of the analysis and visualization functions for multimedia and real-time sensing-data of 5D World Map System has been proposed to make environmental analysis much richer and deeper, which contributes to activities of collaborative environmental knowledge creation [5][6]. Also, a multi-dimensional and multi-layered visualization and Monitoring-Analysis-Warning functions of 5D World Map System for building disaster resilience has been proposed for monitoring Sustainable Development Goals in United Nations ESCAP [8].

The features of the proposed system are its dynamics of dimensional selection and visualization. In the system, every parameter value is expressed in a dimensional way and the correlation calculation is performed in each dimension. The domain of target data, such as a set of healthy cases, a set of disease cases, a set of cases in a specific area, a set of cases with the same age, is also dynamically selected by dimensional selection.

\subsection{A Semantic Visualization Method for Multiple Context of Human Health and Disaster Analysis on 5D World Map System}

In this section, we present "A Clear-Layered Semantic Visualization Method" for multiple context of Human health and disaster analysis on 5D World Map System. As shown in previous section, the 5D World Map System realizes calculating in five dimensions, consisting of three dimensions expressing geographical world map (1st, 2nd, and 3rd dimension), one dimension expressing time (4th dimension), and the 5 th dimension expressing semantics. 
This method focuses on diseases and effects related to disaster cases. This method generates semantic analytical visualization layers as 5th dimension of the 5D World Map System. Our method consists of following steps.

Step-1) Base data (Environmental monitoring data) mapping on 5D World Map System,

Step-2) Upper data (Disease distribution data) mapping on multiple clear layers,

Step-3) Designing combination of base data mapping and upper multiple clear layers according to context of semantic analytical visualization by applying expert knowledge.

Step-1 processes semantic analytical visualization with the functions in 5D World Map System for image, movie, article, and sensor data. Our functions can determine semantic similarities on image and movie data by applying psychological data on color system, and on environmental monitoring data by applying semantic differential computing. Processing of the Step-1 is shown in Figure 3.

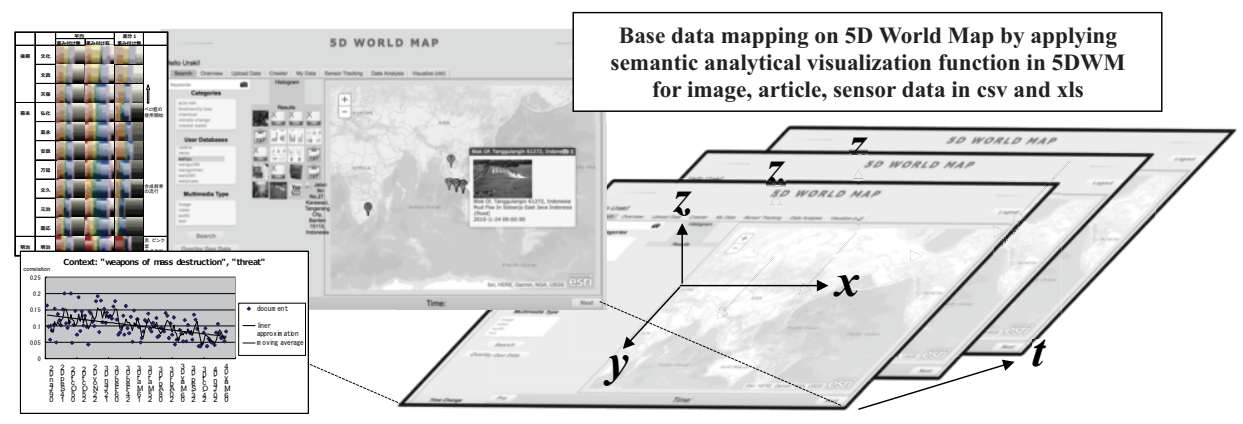

Figure 3. Step-1 Base data (Environmental monitoring data) mapping in 5D World Map System

Step-2 processes upper data mapping on multiple clear layers with followings, 1) to express one semantic in one layer, and 2) the selected layers can be mapped simultaneously (transparency) to integrate/reduce expressions of multiple semantics into one visual dimension as the 5th dimension on the 5D World Map, to make compatibility through multiple contexts and analyses of disaster phenomena. Processing in the Step-2 is shown in Figure 4. 


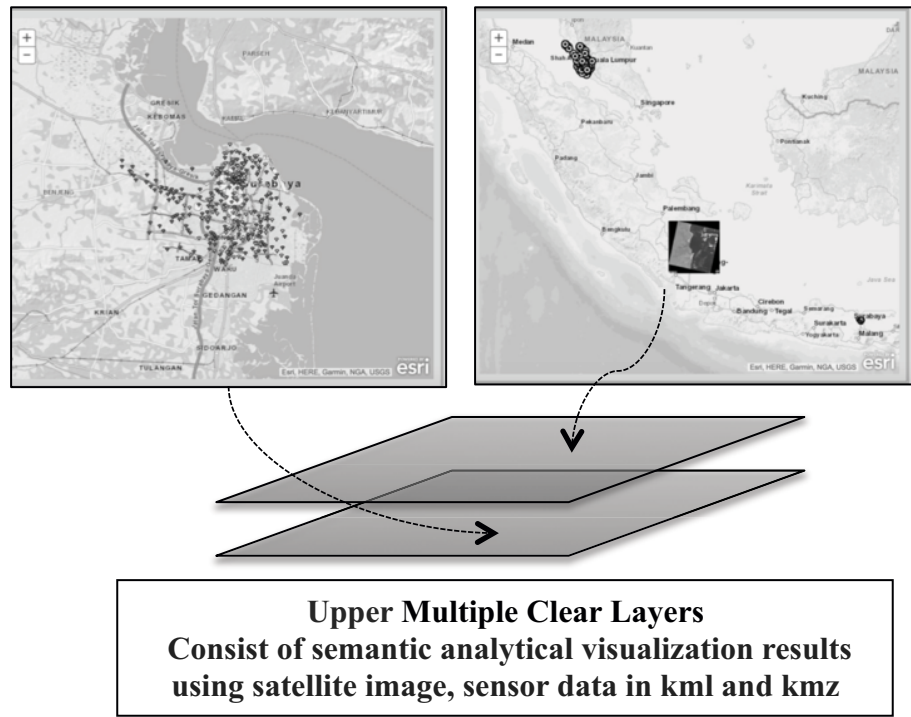

Figure 4. Step-2 Upper data (Disease distribution data) mapping on multiple clear layers

Step-3 designs combinations of base data mapping and upper multiple clear layers according to context of semantic analytical visualization by applying expert knowledge, such as disaster analysis in South Eastern Asia area correspond to SDG11(The SDGs Goal 11 is Sustainable Cities and Communities), which we will show in the next experiment section.

\section{Experiment of A Clear-Layered Semantic Visualization Method for Multiple Context of Disaster Analysis on 5D World Map System}

We realized the experimental system using the SPA functions in 5D World Map and applied the collected multimedia data and environmental monitoring data in South East Asia area. The major and important issues are defined by United Nations as SDGs. We focused on SDG11 as one of the imminent issues for the South East Asia area, and we have implemented two functions in this experiment on 5D World Map. Function-1 is to create a perspective view for disaster, disease, human health, and environmental monitoring data through multiple phenomena and multiple countries, Function- 2 is to create a specific view for disease and human health data.

For the Function-1, Figure 5 shows the analytical results on 5D World Map System to see Human health, disease, and environmental monitoring data from the perspective view through Thailand, Malaysia and Indonesia.

The experiment for Function-1 shows feasibility of our method to see multiple phenomena occurring in multiple countries at once by mapping simultaneously (transparency) to integrate/reduce expressions of multiple semantics into one visual dimension as the 5th dimension on the 5D World Map. This result makes compatibility 
through multiple contexts and analyses of multiple phenomena, such as disaster, disease, human health, and environmental monitoring.

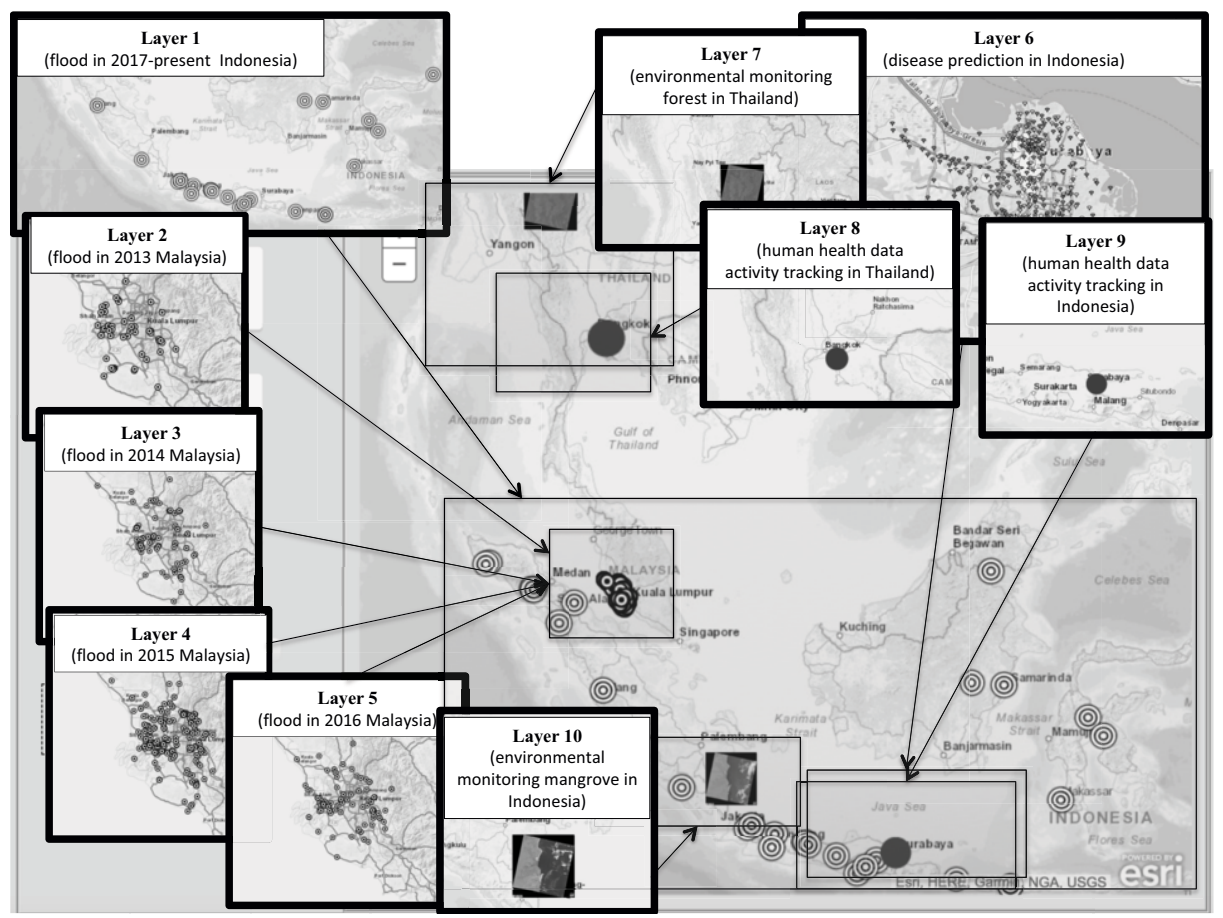

Figure 5. Experimental result for Function-1: Ten upper layers on 5D World Map System to see disaster, disease, human health and environmental monitoring data from the perspective view through Thailand,

Malaysia and Indonesia

For the Function-2, Figure 6 shows the analytical results on 5D World Map System to see specific view in combinatory relationship between disease and human health data in Indonesia.

This result shows, Dengue Fever spreading location and high human activity location at once in the 5D World Map System. Dengue Fever's spreading ways are activities of mosquitos and activities of human, since human can be one of the career of the virus. Therefore, the overlapped place of prevented area and high human activity area can be understood as a place might have the higher risk than the other place.

The experiment for Function-2 shows effectiveness of our method to see multiple phenomena occurring in specific area at once by mapping simultaneously (transparency) to integrate/reduce expressions of multiple semantics into one visual dimension as the 5th dimension on the 5D World Map. This result makes compatibility through disease prevention and human activities to have more meaningful cooccurrence information for people to decrease the disease. 


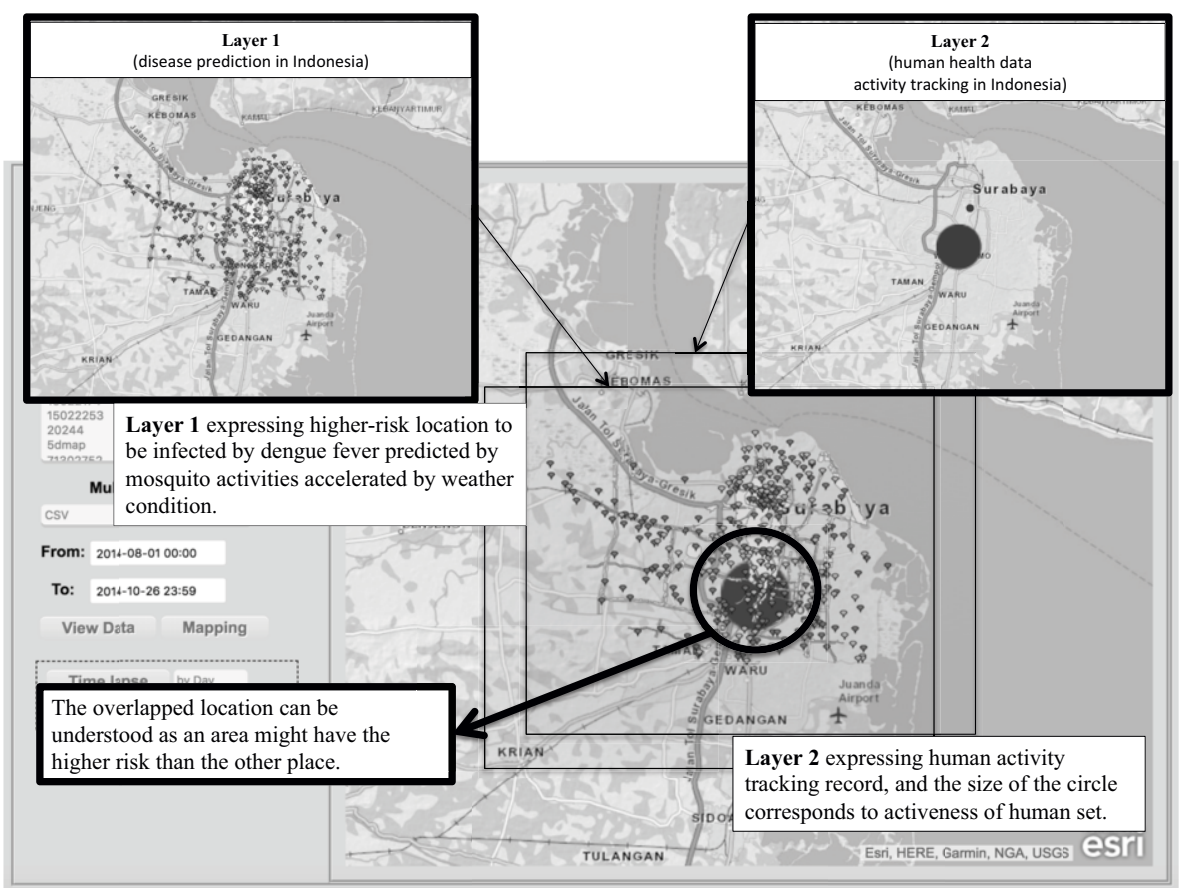

Figure 6. Experimental result for Function-2: Two selected upper layers on 5D World Map System to see specific view in combinatory relationship between disease and human health data in Indonesia.

\section{Conclusion}

We have presented "Human-Health-Analysis Semantic Computing" and "HumanHealth-Analysis Space Creation" for realizing semantic interpretations of situations and symptoms in human-health. This Human-Health-Analysis Semantic Computing method realizes a new multidimensional data analysis and knowledge sharing for a global health monitoring and disease analysis. The computational results are able to be analysed by the time-series difference of the value of each place, the differences between the values of multiple places in a focused area, and the time-series differences between the values of multiple places to detect and predict potential-risk of diseases.

We have applied this method to biological data resources as a new experiment for biological analysis. This system realizes a remote, interactive and real-time global and academic research exchange between remote areas in a global viewpoint. We have also presented Human-Health Computing on the 5D World Map System, as an international and environmental research platform with Spatiotemporal and semantic analysers."

As our future work, we will extend our semantic computing system to new international and collaborative research and education for realizing mutual understanding and knowledge sharing on global human-health issues in the world-wide scope. 


\section{Acknowledgement}

I would like to appreciate Dr. Chalisa Veesommai, Dr. Wajoe Tjatur Sesulihatien and Ms. Yoshiko Itabashi for their significant discussions and experimental studies.

\section{References}

[1] Yasushi Kiyoki, Takashi Kitagawa, Takanari Hayama: "A metadatabase system for semantic image search by a mathematical model of meaning," ACM SIGMOD Record, vol. 23, no. 4, pp.34-41, 1994.

[2] Yasushi Kiyoki, Takashi Kitagawa, Takanari Hayama: "A metadatabase system for semantic image search by a mathematical model of meaning," Multimedia Data Management -- using metadata to integrate and apply digital media--, McGrawHill (book), A. Sheth and W. Klas (editors), Chapter 7, 1998.

[3] Yasushi Kiyoki, Saeko Ishihara: “A Semantic Search Space Integration Method for Meta-level Knowledge Acquisition from Heterogeneous Databases," Information Modeling and Knowledge Bases (IOS Press), Vol. 14, pp.86-103, May 2002.

[4] Yasushi Kiyoki, Shiori Sasaki, Nhung Nguyen Trang, Nguyen Thi Ngoc Diep: "Cross-cultural Multimedia Computing with Impression-based Semantic Spaces," Conceptual Modelling and Its Theoretical Foundations, Lecture Notes in Computer Science, Springer, pp.316-328, March 2012.

[5] Yasushi Kiyoki: "A “Kansei: Multimedia Computing System for Environmental Analysis and CrossCultural Communication," $7^{\text {th }}$ IEEE International Conference on Semantic Computing, keynote speech, Sept. 2013.

[6] Shiori Sasaki, Yusuke Takahashi, Yasushi Kiyoki: "The 4D World Map System with Semantic and Spatiotemporal Analyzers," Information Modelling and Knowledge Bases (IOS Press), Vol.XXI, 18 pages, 2010.

[7] Totok Suhardijanto, Yasushi Kiyoki, Ali Ridho Barakbah: "A Term-based Cross-Cultural Computing System for Cultural Semantics Analysis with Phonological-Semantic Vector Spaces," Information Modelling and Knowledge Bases XXIII, pp.20-38, IOS Press, 2012.

[8] Yasushi Kiyoki, Xing Chen, Shiori Sasaki, Chawan Koopipat: "Multi-Dimensional Semantic Computing with Spatial-Temporal and Semantic Axes for Multi-spectrum Images in Environment Analysis," Information Modelling and Knowledge Bases (IOS Press), Vol. XXVI, pp.14-30, March 2016.

[9] Chalisa Veesommai, Yasushi Kiyoki, Shiori Sasaki, Petchporn Chawakitchareon: "Wide-Area RiverWater Quality Analysis and Visualization with 5D World Map System," Information Modelling and Knowledge Bases (IOS Press), Vol. XXVII, pp.31-41, 2016.

[10] Chalisa Veesommai, Yasushi Kiyoki: "Spatial Dynamics of The Global Water Quality Analysis System with Semantic-Ordering Functions,” Information Modelling and Knowledge Bases, Vol. XXIX, 2018.

[11] Yasushi Kiyoki, Asako Uraki, Chalisa Veesommai: “A Seawater-Quality Analysis Semantic- Space in Hawaii-Islands with Multi- Dimensional World Map System," 18th International Electronics Symposium (IES2016), Bali, Indonesia, September 29-30, 2016.

[12] Shiori Sasaki, Koji Murakami, Yasushi Kiyoki, Asako Uraki: "Global \& Geographical Mapping and Visualization Method for Personal/Collective Health Data with 5D World Map System,” Information Modelling and Knowledge Bases (IOS Press), Vol. XXXII, pp. 134 - 149, 2020.

[13] Shiori Sasaki, Yasushi Kiyoki: "Real-time Sensing, Processing and Actuation Functions of 5D World Map System: A Collaborative Knowledge Sharing System for Environmental Analysis," Information Modelling and Knowledge Bases (IOS Press), Vol. XXVIII, pp. 220-239, May 2016. 